\title{
CONSERVACIÓN DE MASTOFAUNA EN FRAGMENTOS DE BOSQUE CHAQUEÑO EN LA REGIÓN DE MAR CHIQUITA (CÓRDOBA, ARGENTINA).
}

\section{STUDY OF MAMMAL FAUNA IN CHACOAN FOREST FRAGMENTS OF THE MAR CHIQUITA REGION (CÓRDOBA, ARGENTINA) FOR CONSERVATION PURPOSES.}

\author{
Maura Kufner ${ }^{1,2}$, Daniela Tamburini ${ }^{1}$, Liliana Giraudo ${ }^{3}$ y Verónica Briguera ${ }^{1}$
}

\begin{abstract}
Resumen
La reserva Bañados del Río Dulce y Laguna Mar Chiquita (Dpto. Río Seco, Córdoba, Argentina) y zonas aledañas presentan remanentes de Bosque Chaqueño significativos a nivel provincial, que deben conservarse. Se estudió la fauna de mamíferos terrestres y su hábitat en fragmentos dentro (La Cañada) y fuera (Villa Candelaria) del área protegida. El hábitat se describió en base a los estratos de cobertura vegetal. Composición y riqueza estacional de mamíferos se estimaron mediante captura muerta en trampas dispuestas en líneas y por observación directa en recorridos fijos. La estructura vegetal difirió entre localidades, aunque no estacionalmente, probablemente debido al aumento de precipitaciones. Fuera de la reserva el bosque chaqueño presentó una estructura de hábitat más completa (alta cobertura arbustiva y arbórea y dosel superior a $7 \mathrm{~m}$ ). La fauna de mamíferos estuvo representada por 22 especies de las cuales 12 fueron comunes a ambos sitios, aunque la dotación taxonómica y numérica estuvo mejor representada fuera de la reserva. El número de individuos por especie varió estacionalmente. La "pampización” favoreció a los múridos, diferenciando la composición de micromamíferos e influenciando su diversidad que fue mayor en la reserva. Los animales de tamaño mediano estuvieron mejor representados fuera de la misma, la mayoría de éstos vulnerables para la provincia. La composición de la mastofauna denota influencia de las modificaciones antrópicas de su hábitat más que de la típica estacionalidad chaqueña. Los fragmentos de bosque colindantes con la reserva cobran relevancia ante la continua expansión de la superficie agrícola y deberían ser incorporados en el sistema de protección provincial.
\end{abstract}

Palabras claves: mamíferos, bosque chaqueño cordobés, fragmentación, conservación.

\begin{abstract}
Bañados del Río Dulce Laguna Mar Chiquita is a provincial reserve with remnants of Chacoan Forest in its surroundings, that must be conserved. The study was carried out in the Río Seco Department, Córdoba Province, Argentina. Forest fragments were located inside (La Cañada) and outside (Villa Candelaria) the reserve. Habitat descriptors were distribution and cover of vegetation layers. Seasonal composition and richness of mammals in fragments were estimated by dead capture $(\mathrm{CM})$ in 40 traps located along four $100 \mathrm{~m}$ lines, in each location. Mammals directly observed (OD) along fixed transects, were also registered. Vegetation structure differed among localities but not among seasons. Main habitat features in inside fragments were: higher proportion of nude ground, abundant herbaceous covering and absence of strata superior to $7 \mathrm{~m}$. Outside the reserve, high shrub and arboreal cover and canopy over $7 \mathrm{~m}$ high, portray a more typical Chacoan environment. Richness and abundance of mammals were smaller in the reserve (14 species, 2 exclusive; $\mathrm{N}=81$ ) than outside (20 species, 7 exclusive; $\mathrm{N}=179$ ). Twelve common species, over 22, indicated fragment relationships. Similarity was low since outside the reserve, medium sized animals - most vulnerable at the provincial level- reached higher representation. Small mammal composition was different in both localities. Specific diversity of small mammals was higher in the reserve. Modification of habitat to "pampas" favored Murids colonization. Mammal composition denoted influence of human modifications on the habitat, more than that of Chacoan seasonality. Marginal forests collaborate to biological flow, exchanges and relationships that feedback and maintain the biological value of the protected area.
\end{abstract}

Key words: mammals, Córdoba Chacoan forest, fragmentation, conservation.

\section{Introducción}

La riqueza de la mastofauna de los bosques de la región chaqueña cordobesa ha sido mencionada por distintos autores (Bucher \& Ávalos, 1979; Morando \& Polop, 1997; Kufner \& Giraudo, 2001). Actividades humanas sin regulación han provocado reducción de 
este ecosistema a menos del $20 \%$ de su extensión original. Su fragmentación y reemplazo han generado mosaicos de bosques y matorrales secundarios, tierras de cultivo y campos abandonados (Zak \& Cabido, 2002). Esta condición afecta la distribución y diversidad de la fauna, que encuentra en esos relictos de bosque los últimos hábitats chaqueños disponibles a nivel provincial (Chébez, 1994; Kufner \& Giraudo, 2001).

Los Bañados del Río Dulce y Laguna de Mar Chiquita conforman un área bajo protección en la provincia de Córdoba (ACA, 2004). En su zona de influencia cuenta con remanentes significativos de Bosque Chaqueño, que mantendrían flujos, intercambios y relaciones fundamentales para la conservación del ecosistema. De ahí que su protección aumentaría el valor biológico de la reserva a través de incorporación de mayor superficie de hábitat, sitios de biodiversidad, endemismos y especies en riesgo. Este trabajo se propone estudiar la relación entre los parches dentro y fuera del área protegida, en referencia a la fauna de mamíferos terrestres y su hábitat.

\section{Materiales y Métodos}

Área de Estudio: El estudio se llevó a cabo en la margen W de la Reserva de Uso Múltiple Bañados del Río Dulce y Laguna de Mar Chiquita (Dpto. Río Seco, Córdoba), en su zona de influencia comprendida en la llanura chaqueña oriental (Figura 1). El clima es templado; la amplitud térmica es marcada, con temperaturas máxima y mínima absolutas de $-5^{\circ} \mathrm{C}$ y $46^{\circ} \mathrm{C}$ (Capitanelli, 1979). El período lluvioso se extiende de Octubre a Marzo y concentra alrededor del $80 \%$ de las precipitaciones anuales $(580 \mathrm{~mm})$. Si bien la semiaridez y estacionalidad pluvial son características del Chaco, en las últimas décadas existe evidencia de variación climática (INTA, 2002).

Biogeográficamente el área se enclava en el Bosque Chaqueño donde la especie más importante es el quebracho colorado (Schinopsis quebrachocolorado) (Luti et al., 1979). Otros representantes fisonómicos arbóreos y arbustivos destacados son Aspidosperma quebracho-blanco, Celtis tala, Prosopis sp. y Geoffroea decorticans. El bosque aquí se encuentra en aceptable estado de conservación, si bien fragmentado y reducido por uso humano (Cabido \& Zak, 1999). Las actividades económicas principales son la ganadería bovina y la agricultura, en especial de soja.

Metodología:

El estudio se desarrolló en dos localidades de la región: La Cañada y Villa Candelaria, dentro y fuera del área protegida, respectivamente. El muestreo se realizó en dos años consecutivos de condición climática similar (datos del CIM Servicio Meteorológico Nacional, 2005), desde el invierno de 2000 hasta el otoño de 2001 en Villa Candelaria y desde la primavera de 2001 hasta el invierno de 2002 en La Cañada. La investigación se refirió a los períodos seco (invernal) y húmedo (estival).

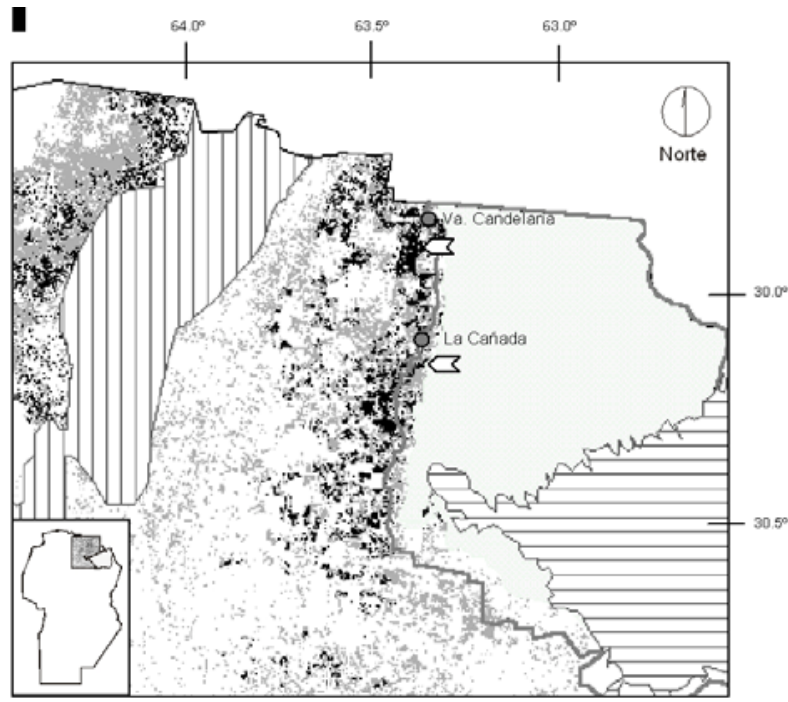

Referencias

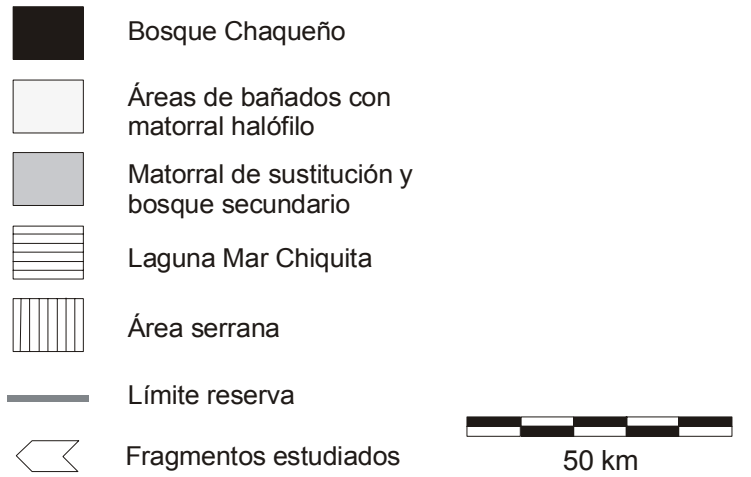

Figura 1. Área de estudio. Bosque chaqueño en el oeste del sistema de Mar Chiquita (modificado de Cabido \& Zak, 1999).

En base a cartografía de la distribución del bosque chaqueño (Cabido \& Zak, 1999), previa verificación de campo mediante un GPS, se establecieron en áreas homogéneas cuatro sitios de muestreo, dos por localidad. La estructura del hábitat se describió a partir de la cobertura vegetal por estratos y la altura del dosel, estimadas según la metodología de Canfield (Hays et al., 1981). Se definieron como muestras mínimas dos transectas fijas de 20 y $30 \mathrm{~m}$ en cada sitio, ocho en total. Para comparar la vegetación de los sitios de estudio, se aplicó el índice Chi Cuadrado de Pearson (Siegel, 1980).

La composición y riqueza de mamíferos pequeños se estimaron en ocho líneas de $100 \mathrm{~m}$, dos por sitio de muestreo, coincidentes con las de vegetación. Cada una constaba de 10 trampas de caída o captura muerta (CM) ubicadas cada $10 \mathrm{~m}$ (80 en total). En cuanto a los mamíferos de tamaño mediano con ámbito de vida 
mayor, se consignaron aquéllos detectados por observación directa (OD) en recorridos fijos equivalentes en ambas localidades. La frecuencia de muestreo fue trimestral en ambos casos.

La composición de la mastofauna se comparó entre ambas localidades mediante el test de máxima verosimilitud $\mathrm{G}^{2}$ (Kent \& Coker, 1992) aplicado al aporte de individuos de las especies comunes, obtenido mediante captura muerta.

La diversidad específica de las comunidades se estimó mediante el índice de diversidad conjunta de Shannon-Wiener $(\mathrm{H})$ y se comparó aplicando el test $\boldsymbol{t}$ de diferencias significativas de la diversidad entre localidades y estaciones (Moreno, 2001). La diversidad de micro y mesomamíferos se analizó en base a los datos de captura muerta y de observación directa, respectivamente.

\section{Resultados}

La condición climática similar considerando los promedios anuales de precipitación durante el estudio $(\boldsymbol{t}=0.35 ; \mathrm{P}=0.731)$ permitió soslayar la discordancia temporal y comparar las variables biológicas entre los campos. Características del hábitat en las localidades de estudio fueron: dentro del área protegida (La Cañada) mayor proporción de suelo desnudo, estrato herbáceo (hasta $50 \mathrm{~cm}$ ) relativamente con mayor cobertura y ausencia de estratos superiores a $7 \mathrm{~m}$. Fuera de la reserva (Villa Candelaria) la cobertura arbustiva y arbórea entre 2 y $3 \mathrm{~m}$, fue mayor y el dosel superó los $7 \mathrm{~m}$ (Tabla 1). La estructura de la vegetación así conformada, difirió entre las localidades $\left(\mathrm{X}^{2}\right.$ Pearson $=36.88 ;$ g.1. $=9$; $\left.\mathrm{P}<0.001\right)$ pero no entre período seco y húmedo fuera ni dentro del área protegida.

En total se registraron 22 especies de mamíferos y 260 individuos a lo largo del estudio. La riqueza de mamíferos y el número de individuos fueron mayores fuera del área protegida (Villa Candelaria: 20 especies y $\mathrm{N}=179$ ) que dentro de la misma (La Cañada: 14 especies y $\mathrm{N}=81$ ) (Tabla 2 ). Doce especies fueron comunes a ambos lugares, de las cuales nueve fueron roedores $(41 \%$ del total).

Fuera de la reserva estuvieron mejor representados los órdenes Didelphimorphia, Cingulata, Carnivora y Rodentia, en general con animales de tamaño mediano y grande. Siete especies fueron registradas sólo aquí: $D$. albiventris, $M$. dimidiata, $C$. vellerosus, $T$. matacus, $C$. chinga, $P$. cancrivorus y $P$. concolor; la mayoría vulnerable para la provincia (Kufner \& Giraudo, 2001). Por su parte, sólo en La Cañada se presentaron dos especies raras (Kufner \& Giraudo, en proceso editorial): $N$. benefactus y $S$. brasiliensis (Tabla 2).

En general, si bien el número de individuos de los micromamíferos fue más abundante en el período seco $(\mathrm{n}=174)$ que en el húmedo $(\mathrm{n}=40)$, la riqueza de especies no acusó variación estacional (Tabla $3 a$ ). Sin embargo, la contribución numérica de las especies difirió entre ambas localidades $\left(\mathrm{MV}-\mathrm{G}^{2}=76.2\right.$; g.1. $=7$; $\mathrm{P}<0.001$ ) (Tabla 3 a y b).

Tabla 1. Cobertura vegetal promedio (\%) por estratos del bosque, dentro (La Cañada) y fuera (Villa Candelaria) de la reserva.

\begin{tabular}{rcc}
\hline $\begin{array}{c}\text { Estrato } \\
\text { (metros) }\end{array}$ & La Cañada & $\begin{array}{c}\text { Villa } \\
\text { candelaria }\end{array}$ \\
\hline $0-0.10$ & 14.873 & 5.836 \\
$0.11-0.50$ & 32.426 & 9.167 \\
$0.51-1.00$ & 7.455 & 10.668 \\
$1.01-2.00$ & 13.654 & 21.107 \\
$2.01-3.00$ & 5.434 & 15.608 \\
$3.01-4.00$ & 8.968 & 6.754 \\
$4.01-5.00$ & 9.849 & 9.284 \\
$5.01-6.00$ & 5.731 & 6.042 \\
$6.01-7.00$ & 1.611 & 4.065 \\
+ de 7.00 & 0.000 & 11.469 \\
\hline
\end{tabular}

Por su parte, los mesomamíferos estuvieron representados por 10 especies en Villa Candelaria y cinco en La Cañada. Durante el período húmedo se registró el doble del número de individuos (Tabla 3b).

La diversidad específica de las comunidades de micromamíferos resultó más elevada dentro que fuera de la reserva (La Cañada $\mathrm{H}=1.722952$, Villa Candelaria $\mathrm{H}=1.035941, \boldsymbol{t}=-5.0289 ; \mathrm{P}<0.001$ ); esta diferencia entre localidades se mantuvo tanto en período seco como húmedo (Tabla 4a). La variación estacional de la diversidad por localidad mostró en La Cañada un índice mayor durante el período húmedo (Tabla 4a); en Villa Candelaria las características de los números no permitieron este análisis. Con respecto a la diversidad de mesomamíferos, no se encontraron diferencias entre ambas localidades (La Cañada $\mathrm{H}$ $=1.459482$ y Villa Candelaria $\mathrm{H}=1.850644)$, ni estacionales (Tabla 4b).

\section{Discusión}

En el Chaco oriental de la provincia la escasa variación de la cobertura de la vegetación entre períodos seco y húmedo se relacionaría con cambio climático en la última década (INTA, 2002), que disminuiría la típica estacionalidad Chaqueña. Por otra parte, el bosque presentó mejor condición fuera de la reserva, manteniéndose la estructura de estratos de vegetación chaqueña característica. En la reserva, el raleo y la tala favorecerían el desarrollo del estrato herbáceo. Es decir que las diferencias halladas en la estructura vegetal de ambos sitios de estudio, serían debidas principalmente a factores antrópicos. 
El uso de diferentes métodos de relevamiento faunístico fue positivo porque permitió un registro diferencial de especies y aumentó las probabilidades de observación.

Tabla 2. Composición de la mastofauna en el Bosque Chaqueño del norte de Córdoba. Número de individuos por especie dentro (La Cañada) y fuera (Villa Candelaria) de la reserva.

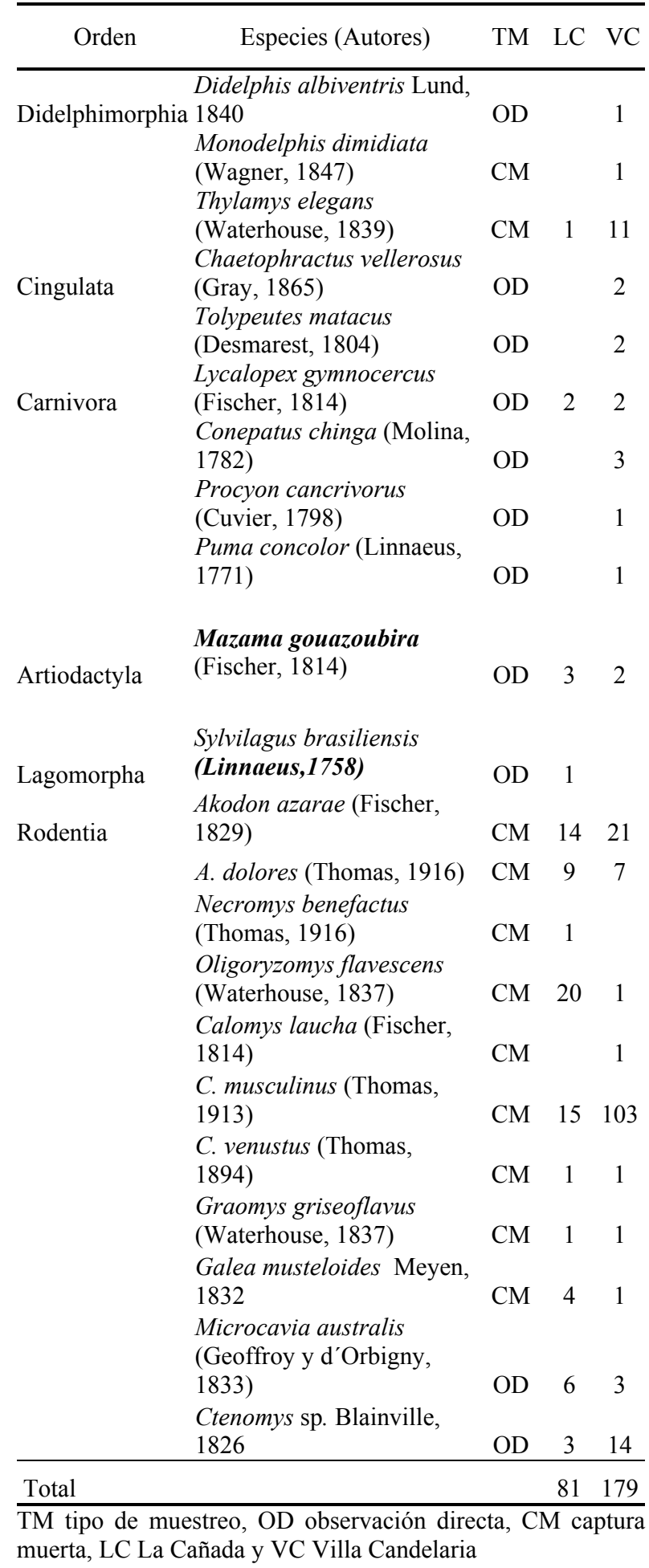

Tabla 3. Contribución estacional de individuos por especie de la mastofauna del Bosque Chaqueño del norte de Córdoba, dentro (La Cañada) y fuera (Villa Candelaria) de la reserva. Referencias: OD observación directa, $\mathrm{CM}$ captura muerta.

a) Micromamíferos (CM)

\begin{tabular}{|c|c|c|c|}
\hline Especie & $\begin{array}{c}\text { La } \\
\text { Cañada }\end{array}$ & $\begin{array}{c}\mathrm{V} . \\
\text { Candelaria }\end{array}$ & Total \\
\hline Thylamys & & & \\
\hline elegans & & 8 & \\
\hline Monodelphys & & & \\
\hline dimidiata & & 1 & \\
\hline Akodon dolores & 6 & 7 & \\
\hline \& Akodon azarae & 6 & 21 & \\
\hline $\begin{array}{l}\text { of Calomys } \\
\text { o }\end{array}$ & 6 & 103 & \\
\hline Calomys & 0 & 100 & \\
\hline 2 venustus & & 1 & \\
\hline Oligoryzomys & & & \\
\hline flavescens & 13 & 1 & \\
\hline Galea & & & \\
\hline musteloides & 1 & & \\
\hline $\mathrm{N}^{\mathrm{o}}$ Individuos & 32 & 142 & 174 \\
\hline $\mathrm{N}^{o}$ Especies & 5 & 7 & 8 \\
\hline Thylamys & & & \\
\hline elegans & 1 & 3 & \\
\hline Akodon dolores & 3 & & \\
\hline $\begin{array}{l}\text { Akodon azarae } \\
\text { Calomys }\end{array}$ & 8 & & \\
\hline musculinus & 9 & & \\
\hline Calomys laucha & & 1 & \\
\hline Calomys & & & \\
\hline $\begin{array}{l}\text { 吾 venustus } \\
\text { Oligoryzomys }\end{array}$ & 1 & & \\
\hline \& flavescens & 7 & & \\
\hline Graomys & & & \\
\hline \& griseoflavus & 1 & 1 & \\
\hline Necromys & & & \\
\hline benefatus & 1 & & \\
\hline Galea & & & \\
\hline musteloides & 3 & 1 & \\
\hline $\mathrm{N}^{\mathrm{o}}$ Individuos & 34 & 6 & 40 \\
\hline $\mathrm{N}^{\circ}$ Especies & 9 & 4 & 10 \\
\hline
\end{tabular}

b)Mesomamíferos (OD)

\begin{tabular}{|c|c|c|c|c|}
\hline \multirow{8}{*}{ 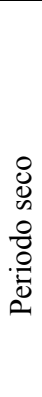 } & Especie & $\begin{array}{c}\text { La } \\
\text { Cañada } \\
\end{array}$ & $\begin{array}{c}\mathrm{V} . \\
\text { Candelaria } \\
\end{array}$ & Total \\
\hline & Didelphis & & 1 & \\
\hline & albiventris & & & \\
\hline & $\begin{array}{l}\text { Microcavia } \\
\text { australis }\end{array}$ & 3 & & \\
\hline & Ctenomys sp. & 1 & & \\
\hline & Chaetophractu & & 2 & \\
\hline & $\begin{array}{l}S \text { vellerosus } \\
\text { Tolypeutes }\end{array}$ & & 2 & \\
\hline & matacus & & & \\
\hline
\end{tabular}


Continuación tabla $3 \ldots$

\begin{tabular}{|c|c|c|c|c|}
\hline & Especie & $\begin{array}{c}\text { La } \\
\text { Cañada }\end{array}$ & $\begin{array}{c}\text { V. } \\
\text { Candelaria }\end{array}$ & Total \\
\hline & Lycalopex & 1 & 2 & \\
\hline & gyтnосегсиs & & & \\
\hline & Conepatus chinga & & 1 & \\
\hline & $\begin{array}{l}\text { Mazama } \\
\text { gouazoubira }\end{array}$ & 1 & 1 & \\
\hline ষ্ঠ & $\mathrm{N}^{\mathrm{o}}$ INDIVIDUOS & 6 & 9 & 15 \\
\hline$\frac{8}{8}$ & $\mathrm{~N}^{\circ}$ ESPECIES & 4 & 6 & 8 \\
\hline 2 & $\begin{array}{l}\text { Microcavia } \\
\text { australis }\end{array}$ & 3 & 3 & \\
\hline & Ctenomys sp. & 2 & 14 & \\
\hline & Lycalopex & 1 & & \\
\hline & gутпосегсиs & & & \\
\hline & Conepatus chinga & & 2 & \\
\hline & Procyon & & 1 & \\
\hline & cancrivorus & & & \\
\hline$\frac{8}{8}$ & Puma concolor & & 1 & \\
\hline 莺 & $\begin{array}{l}\text { Mazama } \\
\text { gouazoubira }\end{array}$ & 2 & 1 & \\
\hline 웅 & Sylvilagus & 1 & & \\
\hline 焉 & brasiliensis & & & \\
\hline & $\mathrm{N}^{\circ}$ INDIVIDUOS & 9 & 22 & 31 \\
\hline & $\mathrm{N}^{\circ}$ ESPECIES & 5 & 6 & 8 \\
\hline
\end{tabular}

Tabla 4. Diversidad específica $(\mathrm{H})$ estacional de mamíferos dentro (La Cañada) y fuera (Villa Candelaria) de la reserva.

a) Micromamíferos (CM)

\begin{tabular}{|c|c|c|c|}
\hline & \multicolumn{3}{|c|}{ Indice $\mathrm{H}$} \\
\hline & La Cañada & Villa Canearia & $t$ \\
\hline $\begin{array}{l}\text { Periodo } \\
\text { seco }\end{array}$ & 1.415861 & 0.930694 & $\begin{array}{l}-3.51444^{*} \\
\text { g.1. }=90.51 \\
0.01>P>0.001\end{array}$ \\
\hline $\begin{array}{l}\text { Periodo } \\
\text { Húmedo }\end{array}$ & 1.860961 & 0.950271 & $\begin{array}{l}-2.66786^{*} \\
\text { g.1. }=7.04 \\
0.05>P>0.02\end{array}$ \\
\hline$t$ & $\begin{array}{l}-2.60538^{*} \\
\text { g.1. }=62.29981 \\
0.02>\mathrm{P}>0.01\end{array}$ & $\begin{array}{l}-0.06002 \\
\text { g.1. }=5.89983 \\
P>0.05\end{array}$ & \\
\hline
\end{tabular}

b) Mesomamíferos (OD)

\begin{tabular}{|c|c|c|c|}
\hline & \multicolumn{3}{|c|}{ Indice $\mathrm{H}$} \\
\hline & La Cañada & Villa Candelaria & $t$ \\
\hline $\begin{array}{l}\text { Periodo } \\
\text { Seco }\end{array}$ & 1.242453 & 1.735126 & $\begin{array}{l}1.342463 \\
\text { g.1. }=11.25 \\
P>0.05\end{array}$ \\
\hline $\begin{array}{l}\text { Periodo } \\
\text { Húmedo }\end{array}$ & 1.522955 & 1.198818 & $\begin{array}{l}1.0465 \\
\text { g.1. }=27.81 \\
P>0.05\end{array}$ \\
\hline$t$ & $\begin{array}{l}-0.76468 \\
\text { g.l. }=11.23926 \\
P>0.05\end{array}$ & $\begin{array}{l}1.73035 \\
\text { g.1. }=27.78311 \\
P>0.05\end{array}$ & \\
\hline
\end{tabular}

OD observación directa y CM captura muerta.
Aunque se constató un 50\% de especies comunes, los bosques externos a la Reserva tuvieron una dotación taxonómica y numéricamente mejor representada, posiblemente en relación con una estructura de hábitat más completa.

El elenco de micromamíferos no varió estacionalmente, probablemente en relación con la escasa variación de la vegetación antes mencionada. Este grupo presentó similar composición específica en ambas localidades, aunque en V. Candelaria la contribución numérica fue superior. En ello influyó $C$. musculinus protagonista de un pico numérico local que incidió además en otros parámetros de la comunidad. Bilenca \& Kravetz (1995) señalan que la proporción de este género en agro-ecosistemas es mayor que en áreas naturales por lo tanto su notable presencia indicaría la influencia de la matriz cultivada. Esta especie pampeana es asimismo reservorio de la fiebre hemorrágica argentina, de epidemiología importante en el país (Polop et al., 1982).

En la composición de la comunidad de micromamíferos de ambas localidades los roedores ocuparon un lugar importante. En ello incidiría la "pampización" antrópica del hábitat merced a la eliminación de estratos intermedios y superiores del bosque y al aumento de cobertura del estrato herbáceo entre 0 y $50 \mathrm{~cm}$. Esta condición del hábitat facilita la colonización por múridos e incide en la diversidad relativamente más alta en La Cañada.

Los mesomamíferos, la mayoría raros y de condición vulnerable en la provincia, estuvieron mejor representados en los bosques externos al área protegida, confiriéndoles mayor valor biológico. Las especies medianas y grandes, con áreas de campeo y requerimientos de hábitat mayores son más susceptibles de extinción, si bien sus amplios rangos de acción facilitarían su movilidad entre parches (Kufner \& Giraudo, 2001). Así, M. australis y M. gouazoubira tuvieron presencia continua en las dos localidades, beneficiadas probablemente el primero por su hábito colonial y el segundo por su especificidad de hábitat (Kufner et al., 1992; Kufner, 2002). Por su parte, S. brasiliensis -con límite austral de distribución en el Chaco cordobés (ACA, 2004)- es una especie sensible que constituye un indicador de buena condición del bosque.

La antropización ha empobrecido la reserva en lo que se refiere a diversidad de la mastofauna, favoreciendo a los roedores y desplazando los mesomamíferos hacia remanentes de bosque vecinos.

En síntesis, los fragmentos de bosque chaqueño en campos privados colindantes con la reserva -debido a su mejor estado de conservación, mayor riqueza de especies e inclusión de taxones vulnerables- deberían ser incorporados en el sistema de protección provincial ya que colaborarían a mantener los flujos e intercambios importantes para la conservación y recuperación de la biodiversidad chaqueña. Estos 
remanentes cobran relevancia ante la continua expansión de la superficie agrícola.

\section{Agradecimientos}

A los directores de las escuelas rurales de Villa Candelaria y La Cañada que nos brindaron albergue y apoyo. A los entonces estudiantes Sobral A., Amelotti I., Crespín I. y Ocampo M. que participaron en el trabajo de campo y laboratorio. Al Centro de Información Meteorológica (CIM) del Servicio Meteorológico Nacional, Fuerza Aérea Argentina, por proporcionar los datos climáticos de la región. A la SECYT de la Universidad Nacional de Córdoba por subsidiar el estudio.

\section{Literatura Citada}

ACA (Agencia Córdoba Ambiente). 2004. Áreas Naturales Protegidas. Provincia de Córdoba. Ediciones el Copista, Córdoba. $122 \mathrm{Pp}$.

Bilenca D. \& F. Kravetz. 1995. Patrones de abundancia relativa en ensambles de pequeños roedores de la región pampeana. Ecología Austral, 5(1): 21-30.

Bucher E.H. \& J.W. Ávalos. 1979. Fauna. 369-434 en: J. Vázquez, R. Miatello \& M. Roque (Eds.). Geografía Física de la Provincia de Córdoba. Ed. Boldt, Buenos Aires. $463 \mathrm{Pp}$.

Cabido M. \& M. Zak. 1999. Vegetación del Norte de Córdoba. Secretaría de Agricultura, Ganadería y Recursos Renovables. Córdoba. 52 Pp.

Capitanelli R.G. 1979. Clima. Pp. 45-138 en: J.B. Vázquez, R. Miatello \& M. Roqué (Eds.). Geografía Física de la Provincia de Córdoba. Ed. Boldt, Buenos Aires. 463 Pp.

Chevez J. 1994. Los que se van. Especies argentinas en peligro. Ed. Albatros. Buenos Aires. $604 \mathrm{Pp}$.

Hays R.L., Summers C. \& W. Seitz. 1981. Estimating wildlife habitat variables. Fish and Wildlife Service USA. FWS/OBS-81/47. $111 \mathrm{Pp}$.

INTA. 2002. El cambio climático y los sistemas deZ producción de la región semiárida-árida central. Jornada de Difusión Técnica. Facultad de Agronomía, UNSL. San Luis, 15 de noviembre de 2002.
Kent M. \& P. Coker. 1992. Vegetation description and análisis. A practical approach. Belhaven Press, London. $363 \mathrm{Pp}$.

Kufner M.B., Sbriller A.P.\& S. Monge. 1992. Relaciones tróficas de una comunidad de herbívoros del desierto del Monte durante la sequía invernal. Iheringia, 72: 112121.

Kufner M.B. \& L. Giraudo. 2001. Distribución de la diversidad y las extinciones de mamíferos de Córdoba en relación con la reducción del bosque y los cambios paleoclimáticos. Boletín de la Academia Nacional de Ciencias, 66: 109-116.

Kufner M.B. 2002. Aportes de los estudios de herbivoría al manejo de ecosistemas áridos y semiáridos del Monte mendocino y el Chaco cordobés. En: Selección de dietas por grandes herbívoros mamíferos (Cid et al. Eds.). Contribuciones del MACN, N 1: 189-191.

Kufner M.B. \& L. Giraudo (Coord.). Los mamíferos de la provincia de Córdoba. Sistemática, corología y estado de conservación. Boletín de la Academia Nacional de Ciencias. En proceso editorial.

Luti R., Beltrán de Solis M.A., Galera F.M., Müller de Ferreira N., Berzal M., Nores M., Herrera M.A. \& J.C. Barrera. 1979. Vegetación. 297-367 en: J. B. Vázquez, R. Miatello y M. Roque (Eds.). Geografía Física de la Provincia de Córdoba. Ed. Boldt, Buenos Aires. 463 Pp.

Morando M. \& J. Polop. 1997. Annotated checklist of mammal species of Córdoba Province, Argentina. Mastozoología Neotropical, 4(2): 129-136.

Moreno C.E. 2001. Métodos para medir la biodiversidad. M\&T- Manuales y Tesis SEA, Volumen I. Zaragoza. $84 \mathrm{Pp}$.

Polop J.J., Cardenal N.C. \& M.S. Sabatini. 1982. Comunidades de roedores en cultivos de sorgo en la Provincia de Córdoba y su posible relación con la Fiebre Hemorrágica Argentina. Ecosur, 9:107-116.

Siegel S. 1980. Estadística no paramétrica aplicada a las ciencias de la conducta. Ed. Trillas, México. 346 Pp.

M. \& M. Cabido. 2002. Spatial patterns of the Chaco vegetation of central Argentina: Integration of remote sensing and phytosociology. Applied Vegetation Science, 5: 213-226.

${ }^{1}$ CONICET, ${ }^{2}$ CERNAR y ${ }^{3}$ Cátedra de Diversidad Animal II, F.C.E.F. y N., U.N.C.

Dirección postal: Vélez Sarsfield 299, 5000, Córdoba, Argentina.

Dirección electrónica: bkufner@com.uncor.edu 